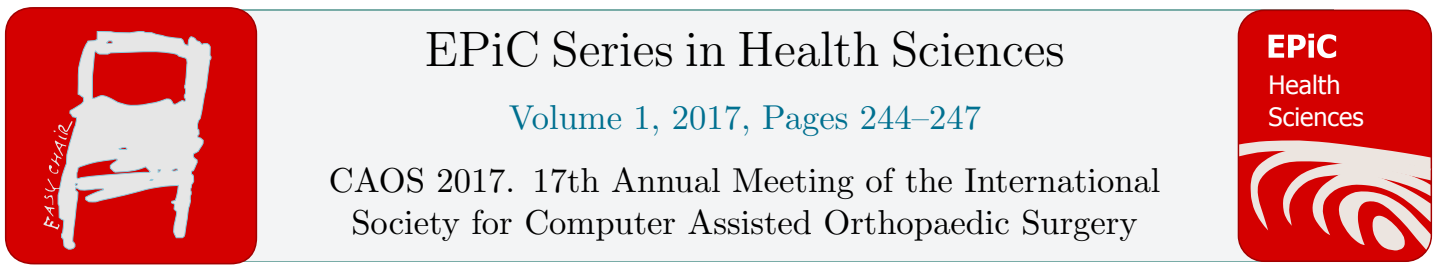

\title{
Precision Orthopaedic Surgery and Precision Orthopaedic Rehabilitation: a novel integrated approach
}

\author{
Paolo Dario ${ }^{1,2}$, Stefano Mazzoleni ${ }^{1}$, Gastone Ciuti $^{1}$, Tao Sun $^{2}$, Zhibin \\ Song ${ }^{2}$, Jian S. Dai ${ }^{3}$ and Shuxin Wang ${ }^{2}$ \\ ${ }^{1}$ The BioRobotics Institute, Scuola Superiore Sant'Anna, Italy \\ ${ }^{2}$ School of Mechanical Engineering, Tianjin University, P. R. China \\ ${ }^{3}$ Centre for Robotics Research, King's College London, UK \\ paolo.dario@santannapisa.it, stefano.mazzoleni@santannapisa.it, \\ gastone.ciuti@santannapisa.it, stao@tju.edu.cn, \\ songzhibin@tju.edu.cn, jian.dai@kcl.ac.uk, shuxinw@tju.edu.cn
}

\begin{abstract}
Orthopaedic surgery is one of the first fields where robotic tools have been used and currently their use is growing thanks to the benefits and advantages of robotics. During the last two decades several robotic devices characterized by high accuracy during both the preoperative and intraoperative phases have been developed. The success of the rehabilitation treatment, which is complementary to the surgical phase, is crucial to the complete recovery of functions: however although computer-assisted navigation is more precise than the conventional techniques, it is still subject to errors. The aim of this study is to present an integrated approach, named "Precision Orthopaedic Surgery Precision Orthopaedic Rehabilitation" (POS-POR), based on new applications for orthopaedic trauma treatment and rehabilitation.
\end{abstract}

\section{Introduction}

Bone fractures represent rather common injuries often requiring surgical treatments. Orthopaedic surgery is one of the first fields where robotic tools have been used and currently their use is growing thanks to the benefits and advantages of robotics (Lang JE, 2011).

Surgical phases, such as the placement on an implant, the reduction of fracture and the cutting or drilling of bone, should be carried out in a very accurate way: the misalignment of the fracture segments is probably the most significant disadvantage because of its high impact on the process of the fracture repair, e.g., development of infections and incomplete recovery of functions. 
Optimal axis alignment correlates with less pain, better knee range of motion, faster rehabilitation, and improved quality of life for patients (Longstaff LM, 2009).

During the last two decades several robotic devices characterized by high accuracy during both the preoperative and intraoperative phases have been developed (Amiot LP, 1995). Currently the scientific literature on the management of long bone fractures shows several studies based on computer-assisted surgical procedures (Matcuk GR, 2016).

The success of the rehabilitation treatment, which is complementary to the surgical phase, is crucial to the complete recovery of functions: however although computer-assisted navigation is more precise than the conventional techniques, it is still subject to errors (Mavrogenis AF, 2013).

The aim of this study is to present an integrated approach based on new applications for orthopaedic trauma treatment and rehabilitation.

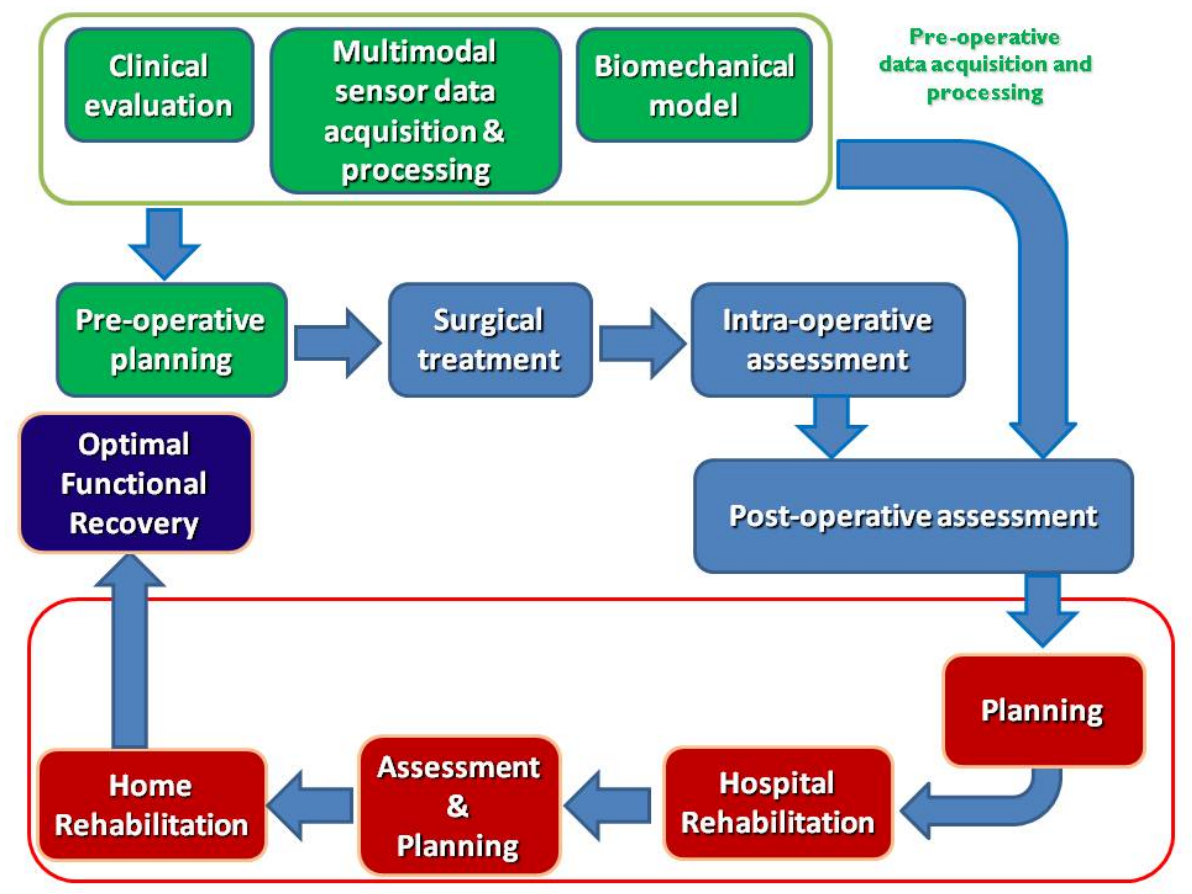

Figure 1: Scheme of the "Precision Orthopaedic Surgery - Precision Orthopaedic Rehabilitation" integrated approach

\section{Material and Methods}

The integrated approach that we are proposing aims to provide a continuity of care from hospital admission to social and work re-entry and it is based on the following phases (Figure 1):

- pre-operative planning based on i) clinical evaluation, ii) multimodal sensor data acquisition and processing and c) an accurate biomechanical model of the joint(s)/segment(s) to be treated;

- surgical treatment (even using robotic devices);

- intra-operative assessment using high-quality online 2D and 3D images; 
- post-operative assessment (using mechanical, ultrasound, electromagnetic, optical techniques,...), where pre-operative data acquisition and processing provide additional inputs;

- precision rehabilitation, where a planning phase based on the outputs of the previous phase provides data and information needed during the hospital rehabilitation (using conventional and robotassisted treatments).

The functional recovery assessment and the subsequent planning are fundamental for the delivery of home-based rehabilitation treatments where serious games and apps can be used for remote monitoring of motor performance. The entire process is intended to lead to an optimal functional recovery and to increase the quality of life of patients.

The name of such integrated approach is "Precision Orthopaedic Surgery - Precision Orthopaedic Rehabilitation" (POS-POR).

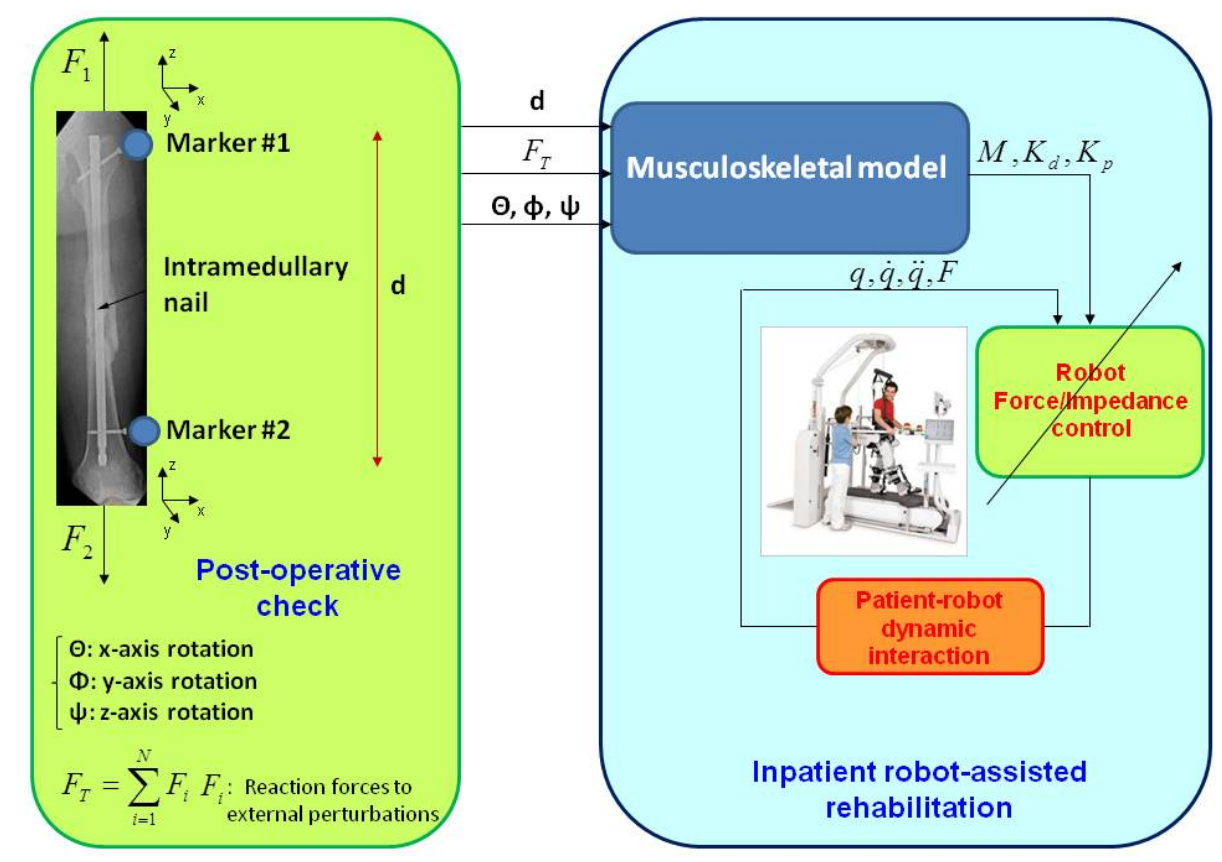

Figure 2: From orthopaedic surgical intervention to orthopaedic rehabilitation.

\section{Results}

The success of the entire process depends heavily on the success of each of the phases. Nonetheless, one crucial aspect is represented by the biomechanical data recorded during the surgical treatment phase representing essential inputs to the musculoskeletal model to be used during the robot-assisted hospital rehabilitation (Figure 2).

The case of long bones fracture treatment represents an example where the presented integrated approach can provide relevant clinical outcomes.

Markers can be used to monitor the distance (d) of distal segments of the bone. This parameter along with the orientation angles around the $\mathrm{x}-, \mathrm{y}$ - and z-axis $(\theta, \varphi, \psi$, respectively) and the reaction 
forces to external perturbations (FT) represent some of the inputs to the musculoskeletal model whose outputs feed the adaptive control scheme of the robotic system (e.g., impedance-based control strategy).

The motion and the forces exerted by the patient who dynamically interacts with the robot during the rehabilitation session represent other inputs to the adaptive control scheme of the robotic system.

\section{Discussion}

The proposed POS-POR integrated approach may provide potential advantages to the achievement of optimal functional recovery that represents the final objective of the entire surgical and rehabilitation orthopaedic process.

Indeed as a consequence of such approach the quality of life of patients who need surgical interventions due to orthopaedic injuries and trauma may increase.

The potential advantages of the application of the POS-POR approach may have an impact in terms of healthcare costs and resources optimization as well. The future works will be focused on the identification of specific clinical cases to be investigated for a validation of the POS-POR approach.

\section{Acknowledgements}

This work has been supported by the Programme of Introducing Talents of Discipline to Universities ("111 Program") under Grant No. B16034 and by the Thousand Talents Program.

\section{References}

Amiot LP, L. H. (1995). Image-guided pedicle screw fixation - a feasibility study. Spine , 20 (10), 1208-1212. 1299 .

Longstaff LM, S. K. (2009). Good alignment after total knee arthroplasty leads to faster rehabilitation and better function. $J$ Arthroplasty, 24 (4), 570-578.

Matcuk GR, M. S. (2016). Stress fractures: pathophysiology, clinical presentation, imaging features, and treatment options. Emerg Radiol, 23 (4), 365-375.

Mavrogenis AF, S. O. (2013). Computer-assisted navigation in orthopedic surgery. Orthopedics, $36(8), 631-642$. 